\title{
Search for top squarks in final states with one lepton targeting pure bino LSP scenarios with the ATLAS detector
}

\author{
David Handl, on behalf of the ATLAS Collaboration \\ Fakultät für Physik, Ludwig-Maximilians-Universität München, Germany \\ E-mail: david.handlecern.ch
}

\begin{abstract}
A search for direct top squark pair production, in final states with one isolated electron or muon, multiple jets and missing transverse momentum, is presented. The analysis is performed using data from proton-proton collisions, which were collected in 2015 and 2016 at a centre-of-mass energy of $\sqrt{s}=13 \mathrm{TeV}$ by the ATLAS collaboration, corresponding to an integrated luminosity of $36.1 \mathrm{fb}^{-1}$.

Only pure bino LSP decay scenarios, where a $\tilde{t}_{1}$ particle decays into a top quark $t$ and a neutralino $\left(\tilde{\chi}_{1}^{0}\right)$ are reported. Additional signatures are considered, including three-body and four-body decays where the top quarks or the $W$ bosons are produced off-shell, depending on the mass splitting between $\tilde{t}_{1}$ and $\tilde{\chi}_{1}^{0}$.

No significant excesses in data compared to the expectations from Standard Model processes are observed, hence the results are interpreted as exclusion limits at 95\% confidence level on the mass of the top squark and the neutralino.
\end{abstract}

EPS-HEP 2017, European Physical Society conference on High Energy Physics 5-12 July 2017

Venice, Italy 


\section{Introduction}

Supersymmetry (SUSY), which extends the Standard Model (SM) by introducing additional particles for every SM degree of freedom, provides an elegant solution to the infamous hierarchy problem. A relatively light top squark (stop) $\tilde{t}_{1}$, the supersymmetric partner of the top quark, is proposed by many theories. Governed by the mass splitting between $\tilde{t}_{1}$ and $\tilde{\chi}_{1}^{0}, \Delta m=m_{\tilde{t}_{1}}-m_{\tilde{\chi}_{1}^{0}}$, only simplified models where a $\tilde{t}_{1}$ decays to a top quark and a neutralino $\left(\tilde{t}_{1} \rightarrow t \tilde{\chi}_{1}^{0}\right)$ are considered. Such decay topologies are referred to as pure bino LSP scenarios. Additionally, three body decays $\left(\tilde{t}_{1} \rightarrow b W \tilde{\chi}_{1}^{0}\right)$ and four body decays $\left(\tilde{t}_{1} \rightarrow b f f^{\prime} \tilde{\chi}_{1}^{0}\right)$, in which the top quark or the $W$ boson are produced off-shell are taken into account [1]. $\tilde{\chi}_{1}^{0}$ is taken to be the lightest supersymmetric particle (LSP), it has no electrical charge and only interacts weakly with ordinary matter, hence it might be a possible candidate for dark matter.

\section{Analysis strategy and background estimation}

The general idea is to extract a phase-space where the SUSY signal is of high purity, denoted as signal region (SR). Remaining SM backgrounds in the signal regions are estimated based on a likelihood fit from data in dedicated control regions (CR), and are further extrapolated to the SR. To evaluate the SM predictions, a series of validation regions (VR) are defined. It is essential that these regions should be similar in terms of kinematics to assure a reliable analysis. Furthermore, two analysis techniques are developed, the 'cut-and-count' method, which is based on counting events in a single SR, and the 'shape-fit' method, where the SR is split into multiple bins in a discriminating variable, enhancing the signal sensitivity, espacially in a phase-space where separation from background is difficult.

To accomplish pure SUSY signatures and to suppress multijet processes, only events with exactly one electron or muon in the final state are selected. In addition, requirements on multiple jets and missing transverse momentum $\left(E_{\mathrm{T}}^{\text {miss }}\right)$ are applied. Events are recorded with a $E_{\mathrm{T}}^{\text {miss }}$ trigger and a requirement on the offline reconstructed $E_{\mathrm{T}}^{\text {miss }}$ above $230 \mathrm{GeV}$ or, if $E_{\mathrm{T}}^{\text {miss }}$ is smaller than the above mentioned threshold, single lepton triggers are applied.

Seven distinct analyses are designed to target a wide range in the $\tilde{t}_{1}-\tilde{\chi}_{1}^{0}$ mass plane. Two signal regions are optimised for medium and high $\tilde{t}_{1}$ masses ( $\mathrm{tN} \_$med, $\mathrm{tN} \_$high). In both regions a requirement is made on a recursively reclustered jet with a mass consistent with $m_{t}$. This is powerful to identify signals, but the approach is also used to design CRs that are either enriched in background processes that contain a hadronically decaying top quark (i.e. $t \bar{t}$ processes) or depleted in such backgrounds (i.e. $W$ boson production).

The diagonal region, where $\Delta m \approx m_{t}$, is very challenging because of the similarity of the kinematics between the signal and the $t \bar{t}$ pair production. These scenarios rely on the presence of high- $p_{\mathrm{T}}$ initial state radiation jets, which serve to boost the di-top squark system. In order to ensure a good separation power in this specific case, three different boosted decision trees (BDT) are implemented and the shape-fit method is employed on the output distribution of the BDTs (tN_diag_low, tN_diag_med, tN_diag_high).

Decays through the three-body channel are possible if $\Delta m$ is smaller than the top quark mass (bWN). The signature is characterised by the shape of the invariant mass of the $b W$ system, hence 
the main discriminative quantity is a variant of the variable $m_{\mathrm{T} 2}$, referred to as $a m_{\mathrm{T} 2}$ [2]. The $a m_{\mathrm{T} 2}$ value peaks at lower values for the signal $(\sim 90 \mathrm{GeV})$, while $t \bar{t}$ events typically saturate at values close to $m_{t}$, thus its distribution is applied to the shape-fit approach.

The mass splitting in the four-body regime is even more compressed, hence final state objects must have lower momenta (bffN). This analysis requires soft leptons with a transverse momentum of $p_{\mathrm{T}} \geq 4 \mathrm{GeV}$, while other analyses require hard leptons of $p_{\mathrm{T}} \geq 25 \mathrm{GeV}$. A shape-fit is performed on the distribution of the ratio of the lepton $p_{\mathrm{T}}$ and $E_{\mathrm{T}}^{\mathrm{miss}}\left(p_{\mathrm{T}}^{\ell} / E_{\mathrm{T}}^{\mathrm{miss}}\right)$ for the model-independent exclusion fit.

\section{Results and Conclusion}

The number of observed events as well as the expected SM prediction in the VRs and SRs for the tN_med, tN_high, bWN and bffN analyses are illustrated in Figure 1 (left). Data and SM expectation are in good agreement within statistical and systematic uncertainties. The three BDT distributions from the $\mathrm{tN}$ _diag analyses are not shown, but also no significant excesses are observed. Exclusion limits at 95\% CL are obtained based on profile-likelihood fits for the signal models. The results extend previous limits by excluding the stop quark mass up to $940 \mathrm{GeV}$ and LSP masses of $300 \mathrm{GeV}$ to $370 \mathrm{GeV}$ (Fig. 1 (right)).
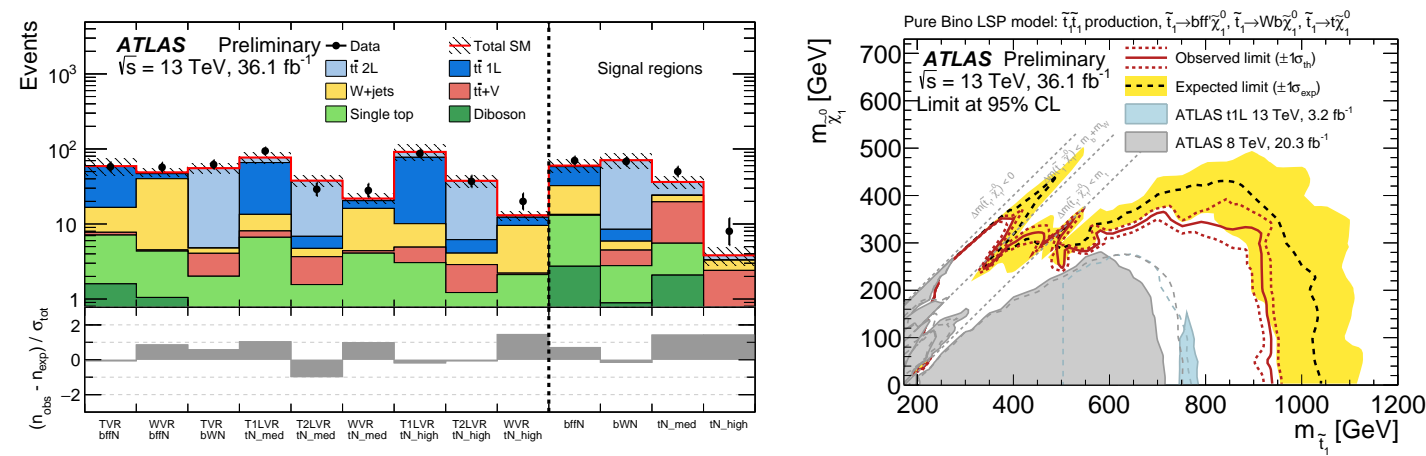

Figure 1: Comparison of observed data with the predicted SM background for the bffN, bWN, tN_med and $\mathrm{tN}$ _high VRs and SRs (left). Expected (black dashed) and observed (red solid) exclusion limit at $95 \%$ confidence level for pure bino LSP models (right) [1].

\section{References}

[1] ATLAS Collaboration, Search for top squark pair production in final states with one lepton, jets and missing transverse momentum using $36.1 \mathrm{fb}^{-1}$ of $\sqrt{s}=13 \mathrm{TeV}$ pp collision data with the ALTAS detector, ATLAS-CONF-2017-37, https://cds.cern.ch/record/2266170.

[2] A. J. Barr, B. Gripaios and C. G. Lester, Transverse masses and kinematic constraints: from the boundary to the crease, JHEP 0911 (2009) 096, arXiv: 0908.3779 [hep-ph] 